\title{
Nociones Fundamentales del Zoo de Partículas Elementales Según el Modelo Estándar
}

\author{
Mgs. Marcel Oswaldo Méndez-Mantuano, \\ Mgs. Evelyn Carolina Egüez Caviedes, \\ Ing. Dayana Shuliet Morales Pastaza, \\ Investigador del Instituto Superior Tecnológico \\ Juan Bautista Aguirre, Ecuador \\ Ing. Simón Bolivar Cruz Muñoz,
} Investigador del Instituto Superior Tecnológico Simón Bolívar, Ecuador

Ing. Erika Maritza Mora Jurado, Investigador de la Unidad Educativa Galo Plaza Lasso

Doi:10.19044/esj.2019.v15n28p124 URL:http://dx.doi.org/10.19044/esj.2019.v15n28p124

\section{Resumen}

La física es una de las ciencias más antiguas, que analiza las interacciones de la materia, la energía, el tiempo y el espacio; con el objetivo de entender el "lenguaje" con el que "habla" el universo. Dentro de este contexto, se encuentran los elementos que conforman el "todo", nos referimos a las partículas elementales (quarks, leptones y bosones), las cuales están organizadas dentro del "modelo estándar de la física de partículas" (coloquialmente conocido como el zoo de partículas), siendo esta una teoría que trata de explicar el funcionamiento del universo, desde las interacciones básicas de la materia, hasta los sistemas complejos como los agujeros negros. Este artículo, lejos de presentarse como una investigación inédita o como un postulado nuevo, trata de abordar un área extensa y compleja, de una manera que sea asimilable para un público interesado en conocer este tema, pero que no desea profundizar en los fundamentos matemáticos que los sostienen. Por lo cual, se realizó una recopilación bibliográfica de diferentes fuentes, en donde se analizan las partículas que conforman el modelo estándar. El objetivo final de esta investigación, es estimular a los lectores a profundizar en el espacio de la física teórica, ya que su entendimiento permite diseñar nuevas estructuras teóricas y tecnológicas en beneficio de la sociedad.

Palabras claves: Bosones, Fermiones, Física, Hadrones, Leptones, Modelo estándar, Partículas elementales, Quarks 


\title{
Fundamental Notions of the Elementary Particle Zoo According to the Standard Model
}

\author{
Mgs. Marcel Oswaldo Méndez-Mantuano, \\ Mgs. Evelyn Carolina Egüez Caviedes, \\ Ing. Dayana Shuliet Morales Pastaza, \\ Investigador del Instituto Superior Tecnológico \\ Juan Bautista Aguirre, Ecuador \\ Ing. Simón Bolivar Cruz Muñoz, \\ Investigador del Instituto Superior Tecnológico Simón Bolívar, Ecuador \\ Ing. Erika Maritza Mora Jurado, \\ Investigador de la Unidad Educativa Galo Plaza Lasso
}

\begin{abstract}
Physics is one of the oldest sciences, which analyzes the interactions of matter, energy, time and space; with the objective of understanding the "language" with which the universe "speaks". Within this context, are the elements that make up the "whole", we refer to elementary particles (quarks, leptons and bosons), which are organized within the "standard model of particle physics" (colloquially known as the particle zoo), this being a theory that tries to explain the functioning of the universe, from the basic interactions of matter, to complex systems such as black holes. This article, far from being presented as an unpublished research or with a new postulate, tries to address a large and complex area, in a way that is assailable to an audience interested in knowing this topic, but who does not want to deepen the mathematical foundations that they hold them. Therefore, a bibliographic compilation of different sources was made, where the particles that make up the standard model are analyzed. The final objective of this research is to stimulate readers to deepen the space of theoretical physics, since their understanding allows designing new theoretical and technological structures for the benefit of society.
\end{abstract}

Keywords: Bosons, Fermions, Physics, Hadrons, Leptons, Standard model, Elementary particles, Quarks 


\section{Introduction}

Hasta hace pocos siglos se consideraba al átomo como algo indivisible, sin embargo, con el pasar de los años estos paradigmas han sido derribados por la supremacía de las pruebas que indican la existencia de otras partículas que forman dicha estructura, la cual está basada en protones, neutrones y electrones, como los miembros más representativos. Durante la segunda mitad del siglo XX, los estudios de física de partículas han cambiado nuestra percepción del mundo, dado que se ha descubierto la existencia de nuevas partículas que interaccionan entre sí, de maneras muy peculiares.

El modelo estándar es una teoría que coincide con las predicciones de la mecánica cuántica y la relatividad especial, la cual se sustenta en la simetría de norma no abeliana (teoría de Yang-Mills) (Cabarcas, 2009). Con este modelo, se puede describir tres de las cuatro fuerzas fundamentales que rigen al universo, como lo son: el electromagnetismo, la fuerza nuclear fuerte (es la responsable de mantener a los quarks unidos creando hadrones), la fuerza nuclear débil (es la fuerza que permite que los neutrones se conviertan en protones, emitiendo radiación en el proceso) y la gravedad, esta última no se encuentra descrita a profundidad por el modelo estándar, ya que su interacción con las otras fuerzas es muy débil y como resultado de ello, no posee efectos medibles a la escala de la física de las partículas elementales, es decir, no presenta actividad en los niveles cuánticos. Este modelo no es perfecto, ya que existen ciertos postulados que no han sido capaces de explicarse en su totalidad, como por ejemplo, el origen de la masa de los neutrinos, o el problema de la jerarquía que involucra al bosón de Higgs, entre otros (Galicia, 2014).

Las cuatro fuerzas fundamentales siguen las siguientes ecuaciones denominadas "el lagrangiano del modelo estándar", las cuales son formulaciones derivadas de las tres leyes de Newton, pero adaptadas a los conceptos de energía, y muy prácticas para las teorías cuánticas de campo de los fermiones y bosones del modelo estándar. Estas fuerzas cuyo grupo de norma es: $G M E=U(1)_{Y} \times S U(2)_{L} \times S U(3)_{C}$, están establecidas por medio de simetrías continuas. Donde se incluye a las interacciones electromagnéticas o $U(1)$, las interacciones de la fuerza nuclear débil $S U(2)$ y las interacciones de la fuerza nuclear fuerte $S U(3)$. La siguiente lagrangiana se obtiene a través del grupo de norma antes mencionado, la cual representa a la energía cinética de los fermiones $\left(L_{k f}\right)$, la energía cinética de los campos de norma $\left(L_{k m}\right)$, el potencial del campo de Higss $\left(\mathcal{L}_{H}\right)$ y el término de los acoplamientos de Yukana $\left(\mathcal{L}_{Y}\right)($ Sánchez, 2014):

$$
\mathcal{L}_{M E}=\mathcal{L}_{k f}+\mathcal{L}_{k m}+\mathcal{L}_{H}+\mathcal{L}_{Y}
$$


Las lagrangianas de cada uno de los términos son las siguientes:

$$
\begin{gathered}
\mathcal{L}_{k f}=\psi i \gamma^{\mu} D_{\mu} \psi \\
\mathcal{L}_{k m}=-1 / 4 F_{\mu v} F^{\mu v}-1 / 4 B_{\mu v} B^{\mu \nu}-1 / 4 G_{\mu v} G^{\mu v} \\
\mathcal{L}_{H}=\left(D^{\mu} \Phi\right)^{f} D_{\mu} \Phi-V\left(\Phi^{\dagger} \Phi\right) \\
\mathcal{L}_{Y}=Y^{e} l_{L} \Phi e_{R}+Y^{u} q_{L} \Phi u_{R}+Y^{d} q_{L} \Phi d_{R}+\text { h.c }
\end{gathered}
$$

Dónde: $\psi i$ es el espinor de Dirac que representa los campos fermiónicos que describen los quarks (y su adjunto de Dirac), $\gamma^{u}$ representa las matrices de Dirac, $D_{u}$ es la derivada covariante asociada al campo gauge gluónico, $F_{u v}$ es el tensor del campo electromagnético, $G_{u v}$ es el tensor de campo gluónico, análogo al tensor campo electromagnético.

Este modelo trata de responder de manera coherente grandes preguntas que se realiza en la actualidad la física moderna, entre las que se pueden incluir al origen de la masa de las partículas. En este aspecto, Peter Higgs en el año de 1964, desarrollo la teoría del "mecanismo de Higgs", el cual trata de explicar el origen de la masa de las partículas, donde sostiene que las masas son proporcionales a la interacción del bosón de Higgs con el campo de Higgs, esto explicaría como ciertas partículas idénticas tienen una masa totalmente diferente (por ejemplo el electrón, el muón y el tauón).

La actual investigación tiene como propósito abordar las diferentes investigaciones que se han realizado en el área de la física de partículas, y mediante un análisis detallar la organización del llamado modelo estándar. Posteriormente se puntualiza en la estructura de los fermiones (quarks y leptones) y cómo estos forman los hadrones, mesones y bariones, y finalmente se especifican a los bosones (fotones, gluones, bosones $\mathrm{W}$ y Z, gravitón y el bosón de Higgs).

\section{Metodología}

El enfoque de la investigación es mixto, ya que se detallan características (de forma cualitativa) de las principales partículas que conforman el modelo estándar, así mismo se indican valores numéricos en diferentes magnitudes que han sido medidas y determinadas. El diseño es documental, dado que la información fue obtenida de diferentes fuentes bibliográficas secundarias (tesis, artículos científicos, etc.), en donde se describen estudios especializados sobre la temática; cabe mencionar que muchos de estos documentos tienen varios años desde su publicación, sin embargo sus afirmaciones no han sido refutadas y permanecen en la actualidad como una fuente fidedigna de consulta. Posee un alcance tanto descriptivo como explicativo, ya que se caracterizaron hechos para establecer un comportamiento y a su vez se establece la relación causa-efecto de varias partículas elementales. 


\section{Desarrollo}

Desde la década de los 70, se estableció al modelo estándar como el gran clasificador de los dos grupos fundamentales que constituyen el universo, en el primero se encuentran los considerados "ladrillos" también denominados fermiones (poseen un espín semientero o fraccionario), y el segundo grupo estan los "pegamentos" o bosones (poseen un espín entero). Los fermiones estan divididos en quarks y leptones, mientras que los bosones se clasifican en bosones de gauge (Universidad de Navarra, 2012).

El modelo estándar menciona 18 tipos básicos de partículas elementales, clasificadas de la siguiente manera:

- 12 fermiones, de los cuales 6 son quarks (arriba, abajo, encanto, extraño, cima y fondo), y 6 son leptones (electrón, muón, tauón, neutrino electrónico, neutrino muónico y neutrino tauónico)

- 6 bosones de gauge (fotón, gluón, bosón W, bosón Z, gluón, el hipotético gravitón y el bosón de Higgs)

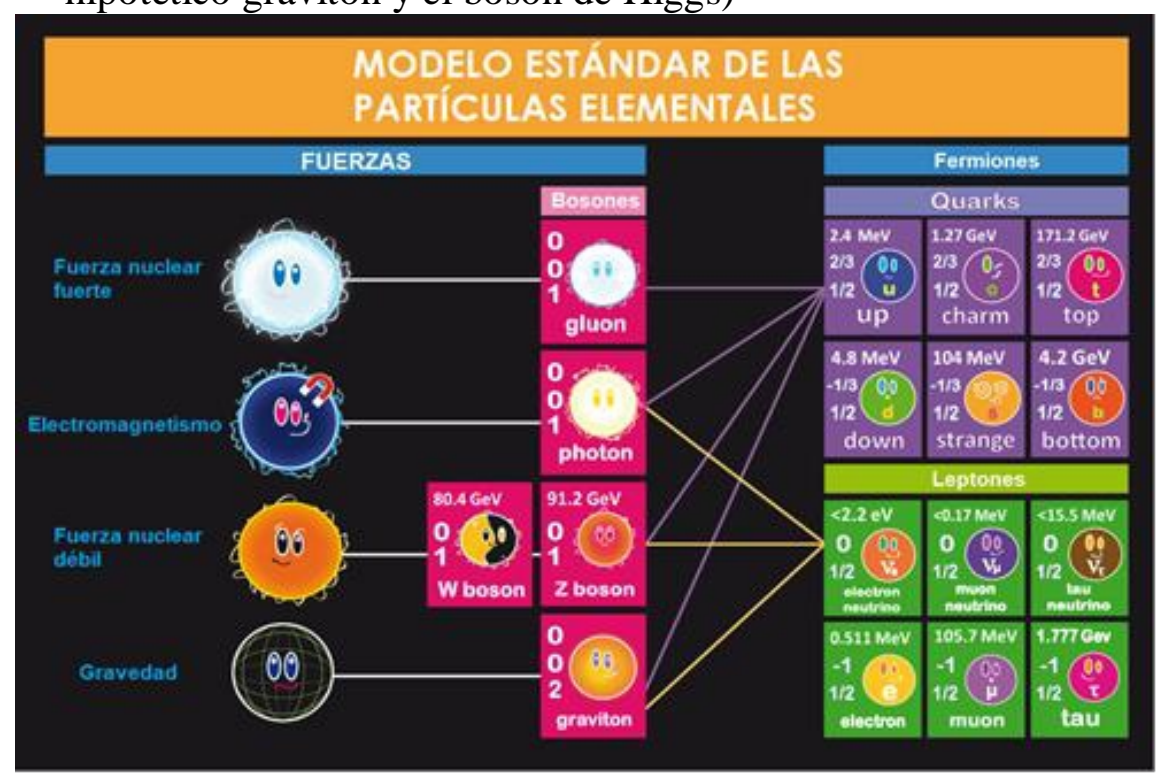

Ilustración 1. Animación de las partículas según el modelo estándar. Fuente: Physicsmore, 2019

Por cada una de las partículas mencionadas, existe una antipartícula propia para estas, que al interaccionar entre ellas se destruyen y generan otras nuevas partículas (Cobián, 2018).

De acuerdo a este modelo, los quarks y leptones son las partículas fundamentales, dado que no poseen una estructura interna; y aquellas partículas que si estan conformadas internamente por otras subpartículas se denominan hadrones o mesones. Los hadrones están constituidos por quarks, y se denominan bariones si están formados por tres quarks o tres antiquarks; 
mientras que los mesones están conformados internamente por un quark y un antiquark; además no se han encontrado quarks libres, sino que estos siempre se encuentran confinados en hadrones o bariones. Los quarks poseen carga eléctrica fraccionaria equivalente a $2 / 3$ y de $1 / 3$, lo cual dependerá del tipo de partícula (Moreira, 2009).

El espín (del inglés spin, en español giro o girar) es denominado como el momento angular intrínseco (como la masa o la carga eléctrica) de las partículas elementales, los cuales están cuantizados, es decir no toman cualquier valor independiente, siendo el espín un valor fijo. Todas las partículas tienen un espín, siendo este una cantidad fija que varía en múltiplos semi enteros $(0,1 / 2,3 / 2,2)$. Las partículas que tiene un espín $1 / 2$, deberán dar dos vueltas completas para llegar a la posición inicial, estas mismas partículas siguen la estadística de Fermi-Dirac (por eso llamada fermiones, los cuales están sujetos al principio de exclusión de Pauli), estas características permiten los arreglos en capas como los átomos. Las partículas asociadas con las fuerzas, tienen un espín entero ( 0 y 1 ) como el fotón o el gluón y siguen la estadística de Bose-Einstein (por eso llamados bosones), y al no tener que repelerse según el principio de exclusión, estas partículas se pueden aglomerar en los mismos estados cuánticos, dando lugar a los condensados de BoseEinstein (Rivas, 2016).

\section{Los quarks}

Como ya fue mencionado anteriormente, existen 6 clases básicas de quarks, también llamados sabores (up, down, strange, charm, bottom, top), y todos ellos poseen carga eléctrica fraccionaria. Los quarks up $(u)$, charm $(c) \mathrm{y}$ top $(t)$, tienen una carga de $+2 / 3$ (lo que significa que la carga del electrón es $2 / 3)$, mientras que los quarks down $(d)$, strange $(s)$ y bottom $(b)$, tienen una carga eléctrica equivalente a $-1 / 3$. Todas las partículas elementales (excepto los neutrinos) interactúan con el electromagnetismo (por su correspondiente carga eléctrica). Los quarks, electrones y neutrinos interaccionan sutilmente con la fuerza nuclear débil y con la gravedad (valor casi nulo), pero únicamente los quarks son sensibles a la fuerza nuclear fuerte. Los quarks denominados pesados (strange, charm, bottom y top), se desintegran en los quarks más ligeros (up y down), por el efecto de la fuerza nuclear débil, efecto similar que tiene la partícula tau que se desintegran con los electrones (Moreira, 2007; Hendry \& Lichtenberg, 1978).

El quark up es el más ligero de ellos, y el quark down es ligeramente más pesado, esto podría justificar la estabilidad que presentan los protones frente a los neutrones. Los protones y neutrones son básicamente la combinación de tres quarks, según la siguiente distribución (Aviléz, 2013):

- Un protón está formado por: dos quarks up y un quark down ( $u u d)$, por ello la carga eléctrica es $+1(2 / 3+2 / 3-1 / 3)$ 
- Un neutrón está formado por: un quark up y dos quarks down ( $u d d)$, por ello la carga eléctrica es $0(2 / 3-1 / 3-1 / 3)$

Cada quark tiene su antipartícula, la cual difiere únicamente en su carga eléctrica correspondiente, siendo esta invertida. Los antiquarks se los representa con una barra horizontal sobre la letra correspondiente al sabor del quark. Las partículas formadas por la combinación par de un quark y un antiquark se las denomina mesones $(q q)$, si están en tríos de quarks se los denomina bariones ( $q q q)$ (Aviléz, 2013).

La estructura de los bariones y mesones es la siguiente (Moreira, 2007):

\begin{tabular}{|c|c|c|c|c|c|}
\hline Bariones & Símbolo & Estructura & Mesones & Símbolo & Estructura \\
\hline Protón & $\mathrm{p}$ & $u u d$ & Pi más & $\pi^{+}$ & $\underline{d} u$ \\
\hline Neutrón & $\mathrm{n}$ & $u d d$ & Pi cero & $\pi^{0}$ & $\underline{u} u / \underline{d} d$ \\
\hline Omega menos & $\Omega^{-}$ & $s s s$ & Pi menos & $\pi^{-}$ & $\underline{u} d$ \\
\hline Sigma más & $\Sigma^{+}$ & $u u s$ & Jota psi & $\mathrm{J} / \psi$ & $\underline{c} c$ \\
\hline Sigma cero & $\Sigma^{0}$ & $u d s$ & Kappa menos & $\kappa^{-}$ & $\underline{u} s$ \\
\hline Sigma menos & $\Sigma^{-}$ & $d d s$ & Kappa cero & $\kappa^{0}$ & $\underline{s} d$ \\
\hline
\end{tabular}

Tabla 1. Estructura de los bariones y mesones

La familia o generación de los quarks, es una agrupación de las partículas de acuerdo a la masa (mayor familia, corresponde a mayor masa), siendo la primera la más ligera y la tercera la más pesada, y no se descarta la existencia de una cuarta familia de ellos (Botella, Branco, Rebelo, \& SilvaMarcos, 2016).

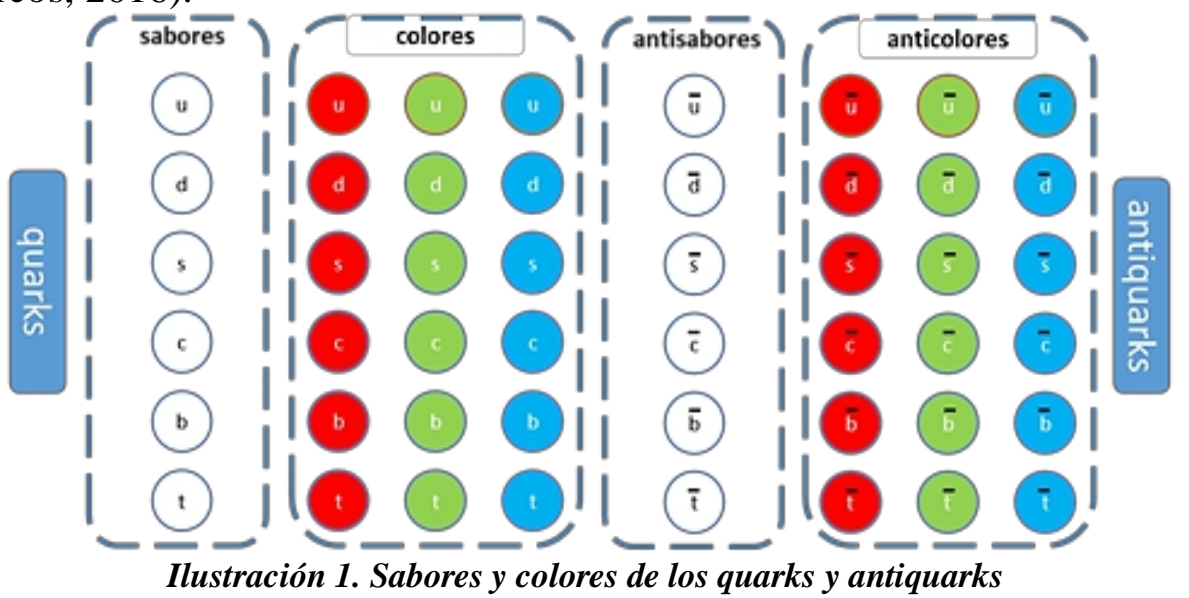

Según el principio de exclusión de Pauli (dos partículas del mismo tipo no pueden ocupar el mismo estado cuántico, estado y espín), tres quarks idénticos no podrían existir, sin embargo el $\Omega$ - es un barión que existente en la amalgama de partículas, el cual tiene tres quarks extraños idénticos (sss). Este problema fue resuelto por el físico Oscar Greenberg, quien propuso que los quarks poseían una propiedad adicional a la cual denominó "carga color" 
(o simplemente color), por ello, existen 36 quarks (ver ilustración 2). Esta propiedad no tiene relación con los efectos de la óptica, sino que es una propiedad análoga a la carga eléctrica, esta última con dos polos conocidos como positivo y negativo, mientras que el color tendría tres formas (rojo, verde, azul). Los quarks poseen colores positivos y los antiquarks poseen colores negativos o anticolores (anti-rojo, anti-verde, anti-azul), definiéndose esta cualidad como una propiedad inherente y exclusiva para los quarks (Moreira, 2007).

Para la determinación de la masa del protón según la QCD (en español Cromodinámica Cuántica), fue definida por los siguientes términos de energía:

$$
H_{Q C D}=H_{m}+H_{q}+H_{g}+H_{a}
$$

El QCD hamiltoniano que refleja la separación masiva de los hadrones, se la puede especificar de la siguiente manera (Silva, 2011):

$$
\begin{gathered}
H_{q}=\int d^{3} x \psi^{+}(-i D . \alpha) \psi \\
H_{m}=\int d^{3} x \psi^{+} m \psi \\
H_{g}=\int d^{3} x \frac{1}{2}\left(E^{2}+B^{2}\right) \\
H_{a}=\int d^{3} x\left[9 \alpha_{S} /(16 \pi)\right]\left(E^{2}-B^{2}\right)
\end{gathered}
$$

Dónde: $H_{q}$ representa a la energía cinética y potencial de los quarks y antiquarks del protón, que contribuyen con una fracción 3(a-b)/4 a la masa del protón, $H_{m}$ es la contribución de la masa de todos los quarks y antiquarks del protón, incluyendo los quarks de valencia, y corresponde a una fracción b de dicha masa, $H_{g}$ representa a la energía cinética y potencial de los gluones del protón y contribuye una fracción 3(1 - a)/4 a la masa total, $H_{a}$ es la energía de los gluones debido a la anomalía de traza, que contribuye con una fracción $(1-b) / 4$ (Villatoro, 2012).

Ji (1995), a partir de la regla de suma de momento inelástica-profunda y la anomalía de traza del tensor de energía-momento, pudo llegar a la deducción de la masa del nucleón, determinándose que 1/8 de la masa de los protones es dada por los quarks y antiquarks $\left(H_{m}\right), 1 / 3$ proviene de la energía cinética y potencial de los mismos quarks y antiquarks $\left(H_{q}\right), 1 / 3$ es dada por la energía cinética y potencial de los gluones $\left(H_{g}\right)$, y un 1/4 corresponde a la anomalía de traza $\left(H_{a}\right)$. Donde únicamente se consideraron tres tipos de quarks (up, down, strange; no se considera el quark charm, ya que el mismo tiene una masa superior a la de los protones), y se realizaron las estimaciones con dos casos extremos para la masa del quark strange:

- Masa pequeña $\left(m_{\mathrm{s}} \rightarrow 0\right)$

- $\operatorname{Masa}$ enorme $\left(\mathrm{m}_{\mathrm{s}} \rightarrow \infty\right)$

Los resultados de estas aproximaciones fueron los siguientes:

\begin{tabular}{|c|c|c|}
\hline Tipo de masa & $\begin{array}{c}\text { ms tiende a 0 } \\
(\mathbf{M e V})\end{array}$ & ms tiende a $\infty(\mathbf{M e V})$ \\
\hline Energía de los quarks (total) & 270 & 300 \\
\hline Masa de los quarks (total) & 160 & 110 \\
\hline
\end{tabular}




\begin{tabular}{|c|c|c|}
\hline Energía de los gluones & 320 & 320 \\
\hline Traza de anomalía & 190 & 210 \\
\hline Total de la masa & $\mathbf{9 4 0}$ & $\mathbf{9 4 0}$ \\
\hline
\end{tabular}

Tabla 2. Aproximaciones de la masa del nucleón

La masa individual de los quarks no ha sido determinada, ya que estos vienen asociados en pares o tríos (no se pueden aislar individualmente), sin embargo varias aproximaciones le dan un valor de $2,4 \mathrm{MeV}$ para el up y 4,8 $\mathrm{MeV}$ para el down, y estimándose en $940 \mathrm{MeV}$ la masa total del nucleón (masa del protón + masa del neutrón). Cabe mencionar que en estos resultados, la proporción del peso de los quarks oscilan entre 11 y $17 \%$, los cuales han sido corroborados por investigadores como Silva (2011); pero otro grupo de investigadores (como Meyer, 2011), le dan una proporción inferior al $9 \%$ del peso total del nucleón.

Se puede resumir que los quarks tienen las siguientes características:

\begin{tabular}{|c|c|c|c|c|c|}
\hline $\begin{array}{c}\text { Nombre en } \\
\text { español }\end{array}$ & $\begin{array}{c}\text { Nombre en } \\
\text { inglés }\end{array}$ & Familia & Masa $\left(\mathbf{M e V / c ^ { 2 }}\right)$ & Carga eléctrica (e) & Espín \\
\hline Arriba & Up $(u)$ & Primera & $1,5-4,0$ & $+2 / 3$ & $+1 / 2$ \\
\hline Abajo & Down $(d)$ & Primera & $4-8$ & $-1 / 3$ & $-1 / 2$ \\
\hline Extraño & Strange $(s)$ & Segunda & $80-130$ & $-1 / 3$ & $-1 / 2$ \\
\hline Encantado & Charm $(c)$ & Segunda & $1150-1350$ & $+2 / 3$ & $+1 / 2$ \\
\hline Fondo & Bottom $(b)$ & Tercera & $4100-4400$ & $-1 / 3$ & $+1 / 2$ \\
\hline Cima & Top $(t)$ & Tercera & 170900 & $+2 / 3$ & $+1 / 2$ \\
\hline
\end{tabular}

Tabla 3. Detalle de las diferentes características de los quarks. Fuente: Amsler, DeGrand, \& Krusche, 2018

\section{Los leptones}

En 1936 el científico Carl Anderson descubre el muón $(\mu)$, el cual tiene características similares a las del electrón, difiriendo principalmente en su masa, siendo este 210 veces más grande que el electrón. Para el año de 1975 Martin Perl, descubre la partícula del tau $(\tau)$, la cual es 4000 veces la masa del electrón. El nombre de las partículas fue acuñado por León Rosenfeld, a las cuales denominó leptones, proveniente del griego "leptos", que significa ligero o delgado (Gelmeni, 2014).

Para muchos, los leptones son partículas verdaderamente fundamentales, ya que no poseen una estructura interna (aparentemente). Igual que los quarks, los leptones poseen un espín fraccionario equivalente a 1/2, que pueden tener carga eléctrica o no (como el caso de los neutrinos). Los principales leptones encontrados experimentalmente son: el electrón, el muón, el tau, y los tres neutrinos (neutrino del electrón, neutrino del muón y el neutrino del tau), siendo el primero el más representativo de todos ellos (Moreira, 2007). 
Se conoce que los leptones son partículas susceptibles a la interacción con la fuerza nuclear débil y el electromagnetismo, sin embargo, no se ha podido determinar que sean capaces de interactuar con la fuerza nuclear fuerte; pero esto, no significa que se excluya esta posibilidad (Ordoñéz, 1992).

Los leptones son fermiones que no poseen carga hadrónica o de color. Los leptones también están separados en tres sabores, los cuales son: el leptón electrón, el leptón muón y el leptón tau; donde en cada sabor existen dos partículas designadas como "doblete débil". La primera es una partícula con carga masiva, que lleva el nombre del sabor correspondiente (electrón, muón, tau) y la otra es una partícula con carga eléctrica neutra, con una masa extremadamente pequeña, llamada neutrinos (neutrino electrónico, neutrino del muón, nutrino del tau). Cada partícula leptónica tiene su constituyente de antipartículas, las cuales son: el positrón, antimuón, antitauón, antineutrino electrónico, antineutrino del muón y el antineutrino del tau. Estas partículas están agrupadas en tres familias o generaciones (igual que los quarks), es decir, poseen similares interacciones, pero difieren en su masa, donde las primeras familias son las más ligeras y las posteriores son las más pesadas; por ejemplo, el electrón, el muón y el tau poseen los mismos números cuánticos, pero sus masas son radicalmente diferentes (Mileo, 2017).

El resumen de las características de los leptones es la siguiente:

\begin{tabular}{|c|c|c|c|c|c|}
\hline Nombre & Símbolo & Familia & $\begin{array}{c}\text { Masa } \\
\left(\mathbf{M e V / \mathbf { c } ^ { 2 }}\right)\end{array}$ & $\begin{array}{c}\text { Carga } \\
\text { eléctrica }(\boldsymbol{e})\end{array}$ & Espín \\
\hline Electrón & $e$ & Primera & 0,511 & -1 & $1 / 2$ \\
\hline $\begin{array}{c}\text { Neutrino del } \\
\text { electrón } \\
\text { (electrónico) }\end{array}$ & $V e$ & Primera & $<0,0000022$ & 0 & $1 / 2$ \\
\hline Muón & $\mu$ & Segunda & 105,7 & -1 & $1 / 2$ \\
\hline $\begin{array}{c}\text { Neutrino del } \\
\text { muón }\end{array}$ & $V \mu$ & Segunda & $<0,17$ & 0 & $1 / 2$ \\
\hline Tau & $\tau$ & Tercera & 1777 & -1 & $1 / 2$ \\
\hline $\begin{array}{c}\text { Neutrino del } \\
\text { tau }\end{array}$ & $V \tau$ & Tercera & $<15,5$ & 0 & $1 / 2$ \\
\hline
\end{tabular}

Tabla 1. Detalle de las diferentes características de los leptones

Según el modelo clásico de Bohr, los electrones están definidos en términos de su posición y de su energía. Los cuales giran alrededor del núcleo atómico en orbitales discretos, cuya posición de un electrón particular se define en términos de su orbital, donde la energía esta cuantizada, en otras palabras, los electrones solo pueden tener valores específicos de energía, para cambiar estos valores, deberán realizar saltos cuánticos a valores permitidos (niveles superiores absorben energía; inferiores emiten energía). Los estados electrónicos están determinados según el principio de exclusión de Pauli, donde se establece que cada estado únicamente puede estar ocupado por dos electrones que deben tener espines opuestos. Por ello, los niveles atómicos $s$, 
$p, d$ y $f$ pueden agrupar un total de 2, 6, 10 y 14 electrones respectivamente; sin embargo, no todos los niveles se encuentran completos en un determinado átomo. Esto indica que los electrones son quienes dan la valencia a los diferentes elementos químicos, donde la capas más externas son las que permiten los encales entre átomos, de los compuestos y moléculas, además muchas de las características de los sólidos, está determinada por las configuraciones electrónicas de las valencias (Callister, 2007).

Los principales elementos químicos poseen las siguientes configuraciones electrónicas:

\begin{tabular}{|c|c|c|c|}
\hline $\mathbf{Z}$ & Elemento & Símbolo & Configuración electrónica \\
\hline 1 & Hidrógeno & $\mathrm{H}$ & $1 \mathrm{~s}^{1}$ \\
\hline 2 & Helio & $\mathrm{He}$ & $1 \mathrm{~s}^{2}$ \\
\hline 3 & Litio & $\mathrm{Li}$ & $1 \mathrm{~s}^{2} 2 \mathrm{~s}^{1}$ \\
\hline 4 & Berilio & $\mathrm{Be}$ & $1 \mathrm{~s}^{2} 2 \mathrm{~s}^{2}$ \\
\hline 5 & Boro & B & $1 s^{2} 2 s^{2} 2 p^{1}$ \\
\hline 6 & Carbono & $\mathrm{C}$ & $1 \mathrm{~s}^{2} 2 \mathrm{~s}^{2} 2 \mathrm{p}^{2}$ \\
\hline 7 & Nitrógeno & $\mathrm{N}$ & $1 s^{2} 2 s^{2} 2 p^{3}$ \\
\hline 8 & Oxígeno & $\mathrm{O}$ & $1 \mathrm{~s}^{2} 2 \mathrm{~s}^{2} 2 \mathrm{p}^{4}$ \\
\hline 9 & Flúor & $\mathrm{F}$ & $1 s^{2} 2 s^{2} 2 p^{5}$ \\
\hline 10 & Neón & $\mathrm{Ne}$ & $1 s^{2} 2 s^{2} 2 p^{6}$ \\
\hline 11 & Sodio & $\mathrm{Na}$ & $1 s^{2} 2 s^{2} 2 p^{6} 3 s^{1}$ \\
\hline 12 & Magnesio & $\mathrm{Mg}$ & $1 s^{2} 2 s^{2} 2 p^{6} 3 s^{2}$ \\
\hline 13 & Aluminio & $\mathrm{Al}$ & $1 s^{2} 2 s^{2} 2 p^{6} 3 s^{2} 3 p^{1}$ \\
\hline 14 & Silicio & $\mathrm{Si}$ & $1 s^{2} 2 s^{2} 2 p^{6} 3 s^{2} 3 p^{2}$ \\
\hline 15 & Fósforo & $P$ & $1 s^{2} 2 s^{2} 2 p^{6} 3 s^{2} 3 p^{3}$ \\
\hline 16 & Azufre & $\mathrm{S}$ & $1 s^{2} 2 s^{2} 2 p^{6} 3 s^{2} 3 p^{4}$ \\
\hline 17 & Cloro & $\mathrm{Cl}$ & $1 s^{2} 2 s^{2} 2 p^{6} 3 s^{2} 3 p^{5}$ \\
\hline 18 & Argón & $\mathrm{Ar}$ & $1 s^{2} 2 s^{2} 2 p^{6} 3 s^{2} 3 p^{6}$ \\
\hline 19 & Potasio & $\mathrm{K}$ & $1 s^{2} 2 s^{2} 2 p^{6} 3 s^{2} 3 p^{6} 4 s^{1}$ \\
\hline 20 & Calcio & $\mathrm{Ca}$ & $1 s^{2} 2 s^{2} 2 p^{6} 3 s^{2} 3 p^{6} 4 s^{2}$ \\
\hline 21 & Escandio & $\mathrm{Sc}$ & $1 s^{2} 2 s^{2} 2 p^{6} 3 s^{2} 3 p^{6} 3 d^{1} 4 s^{2}$ \\
\hline 22 & Titanio & $\mathrm{Ti}$ & $1 s^{2} 2 s^{2} 2 p^{6} 3 s^{2} 3 p^{6} 3 d^{2} 4 s^{2}$ \\
\hline 23 & Vanadio & $\mathrm{V}$ & $1 s^{2} 2 s^{2} 2 p^{6} 3 s^{2} 3 p^{6} 3 d^{3} 4 s^{2}$ \\
\hline 24 & Cromo & $\mathrm{C}$ & $1 s^{2} 2 s^{2} 2 p^{6} 3 s^{2} 3 p^{6} 3 d^{5} 4 s^{1}$ \\
\hline 25 & Manganeso & $\mathrm{Mn}$ & $1 s^{2} 2 s^{2} 2 p^{6} 3 s^{2} 3 p^{6} 3 d^{5} 4 s^{2}$ \\
\hline 26 & Hierro & $\mathrm{Fe}$ & $1 s^{2} 2 s^{2} 2 p^{6} 3 s^{2} 3 p^{6} 3 d^{6} 4 s^{2}$ \\
\hline 27 & Cobalto & Co & $1 s^{2} 2 s^{2} 2 p^{6} 3 s^{2} 3 p^{6} 3 d^{7} 4 s^{2}$ \\
\hline 28 & Níquel & $\mathrm{Ni}$ & $1 s^{2} 2 s^{2} 2 p^{6} 3 s^{2} 3 p^{6} 3 d^{8} 4 s^{2}$ \\
\hline 29 & Cobre & $\mathrm{Cu}$ & $1 s^{2} 2 s^{2} 2 p^{6} 3 s^{2} 3 p^{6} 3 d^{10} 4 s^{1}$ \\
\hline 30 & Cinc & $\mathrm{Zn}$ & $1 s^{2} 2 s^{2} 2 p^{6} 3 s^{2} 3 p^{6} 3 d^{10} 4 s^{2}$ \\
\hline 31 & Galio & $\mathrm{Ga}$ & $1 s^{2} 2 s^{2} 2 p^{6} 3 s^{2} 3 p^{6} 3 d^{10} 4 s^{2} 4 p^{1}$ \\
\hline 32 & Germanio & $\mathrm{Ge}$ & $1 s^{2} 2 s^{2} 2 p^{6} 3 s^{2} 3 p^{6} 3 d^{10} 4 s^{2} 4 p^{2}$ \\
\hline 33 & Arsénico & As & $1 s^{2} 2 s^{2} 2 p^{6} 3 s^{2} 3 p^{6} 3 d^{10} 4 s^{2} 4 p^{3}$ \\
\hline 34 & Selenio & $\mathrm{Se}$ & $1 s^{2} 2 s^{2} 2 p^{6} 3 s^{2} 3 p^{6} 3 d^{10} 4 s^{2} 4 p^{4}$ \\
\hline 35 & Bromo & $\mathrm{Br}$ & $1 s^{2} 2 s^{2} 2 p^{6} 3 s^{2} 3 p^{6} 3 d^{10} 4 s^{2} 4 p^{5}$ \\
\hline 36 & Kriptón & $\mathrm{Kr}$ & $1 s^{2} 2 s^{2} 2 p^{6} 3 s^{2} 3 p^{6} 3 d^{10} 4 s^{2} 4 p^{6}$ \\
\hline 37 & Rubidio & $\mathrm{Rb}$ & $1 s^{2} 2 s^{2} 2 p^{6} 3 s^{2} 3 p^{6} 3 d^{10} 4 s^{2} 4 p^{6} 5 s^{1}$ \\
\hline
\end{tabular}




\begin{tabular}{|c|c|c|c|}
\hline 38 & Estroncio & $\mathrm{Sr}$ & $1 s^{2} 2 s^{2} 2 p^{6} 3 s^{2} 3 p^{6} 3 d^{10} 4 s^{2} 4 p^{6} 5 s^{2}$ \\
\hline 39 & Itrio & $\mathrm{Y}$ & $1 s^{2} 2 s^{2} 2 p^{6} 3 s^{2} 3 p^{6} 3 d^{10} 4 s^{2} 4 p^{6} 4 d^{1} 5 s^{2}$ \\
\hline 40 & Circonio & $\mathrm{Zr}$ & $1 s^{2} 2 s^{2} 2 p^{6} 3 s^{2} 3 p^{6} 3 d^{10} 4 s^{2} 4 p^{6} 4 d^{2} 5 s^{2}$ \\
\hline 41 & Niobio & $\mathrm{Nb}$ & $1 s^{2} 2 s^{2} 2 p^{6} 3 s^{2} 3 p^{6} 3 d^{10} 4 s^{2} 4 p^{6} 4 d^{4} 5 s^{1}$ \\
\hline 42 & Molibdeno & Mo & $1 s^{2} 2 s^{2} 2 p^{6} 3 s^{2} 3 p^{6} 3 d^{10} 4 s^{2} 4 p^{6} 4 d^{5} 5 s^{1}$ \\
\hline 43 & Tecnecio & Tc & $1 s^{2} 2 s^{2} 2 p^{6} 3 s^{2} 3 p^{6} 3 d^{10} 4 s^{2} 4 p^{6} 4 d^{5} 5 s^{2}$ \\
\hline 44 & Rutenio & $\mathrm{Ru}$ & $1 s^{2} 2 s^{2} 2 p^{6} 3 s^{2} 3 p^{6} 3 d^{10} 4 s^{2} 4 p^{6} 4 d^{7} 5 s^{1}$ \\
\hline 45 & Rodio & $\mathrm{Rh}$ & $1 s^{2} 2 s^{2} 2 p^{6} 3 s^{2} 3 p^{6} 3 d^{10} 4 s^{2} 4 p^{6} 4 d^{8} 5 s^{1}$ \\
\hline 46 & Paladio & $\mathrm{Pd}$ & $1 s^{2} 2 s^{2} 2 p^{6} 3 s^{2} 3 p^{6} 3 d^{10} 4 s^{2} 4 p^{6} 4 d^{10}$ \\
\hline 47 & Plata & $\mathrm{Ag}$ & $1 s^{2} 2 s^{2} 2 p^{6} 3 s^{2} 3 p^{6} 3 d^{10} 4 s^{2} 4 p^{6} 4 d^{10} 5 s^{1}$ \\
\hline 48 & Cadmio & $\mathrm{Cd}$ & $1 s^{2} 2 s^{2} 2 p^{6} 3 s^{2} 3 p^{6} 3 d^{10} 4 s^{2} 4 p^{6} 4 d^{10} 5 s^{2}$ \\
\hline 49 & Indio & In & $1 s^{2} 2 s^{2} 2 p^{6} 3 s^{2} 3 p^{6} 3 d^{10} 4 s^{2} 4 p^{6} 4 d^{10} 5 s^{2} 5 p^{1}$ \\
\hline 50 & Estaño & $\mathrm{Sn}$ & $1 s^{2} 2 s^{2} 2 p^{6} 3 s^{2} 3 p^{6} 3 d^{10} 4 s^{2} 4 p^{6} 4 d^{10} 5 s^{2} 5 p^{2}$ \\
\hline 51 & Antimonio & $\mathrm{Sb}$ & $1 s^{2} 2 s^{2} 2 p^{6} 3 s^{2} 3 p^{6} 3 d^{10} 4 s^{2} 4 p^{6} 4 d^{10} 5 s^{2} 5 p^{3}$ \\
\hline 52 & Telurio & $\mathrm{Te}$ & $1 s^{2} 2 s^{2} 2 p^{6} 3 s^{2} 3 p^{6} 3 d^{10} 4 s^{2} 4 p^{6} 4 d^{10} 5 s^{2} 5 p^{4}$ \\
\hline 53 & Yodo & $\mathrm{I}$ & $1 s^{2} 2 s^{2} 2 p^{6} 3 s^{2} 3 p^{6} 3 d^{10} 4 s^{2} 4 p^{6} 4 d^{10} 5 s^{2} 5 p^{5}$ \\
\hline 54 & Xenón & $\mathrm{Xe}$ & $1 s^{2} 2 s^{2} 2 p^{6} 3 s^{2} 3 p^{6} 3 d^{10} 4 s^{2} 4 p^{6} 4 d^{10} 5 s^{2} 5 p^{6}$ \\
\hline 55 & Cesio & $\mathrm{Cs}$ & {$[\mathrm{Xe}] 6 \mathrm{~s}^{1}$} \\
\hline 56 & Bario & $\mathrm{Ba}$ & {$[\mathrm{Xe}] 6 \mathrm{~s}^{2}$} \\
\hline 57 & Lantano & $\mathrm{La}$ & {$[\mathrm{Xe}] 5 \mathrm{~d}^{1} 6 \mathrm{~s}^{2}$} \\
\hline 72 & Hafnio & Hf & {$[\mathrm{Xe}] 4 \mathrm{f}^{14} 5 \mathrm{~d}^{2} 6 \mathrm{~s}^{2}$} \\
\hline 73 & Tantalio & $\mathrm{Ta}$ & {$[\mathrm{Xe}] 4 \mathrm{f}^{14} 5 \mathrm{~d}^{3} 6 \mathrm{~s}^{2}$} \\
\hline 74 & Volframio & $\mathrm{W}$ & {$[\mathrm{Xe}] 4 \mathrm{f}^{14} 5 \mathrm{~d}^{4} 6 \mathrm{~s}^{2}$} \\
\hline 75 & Renio & $\operatorname{Re}$ & {$[\mathrm{Xe}] 4 \mathrm{f}^{14} 5 \mathrm{~d}^{5} 6 \mathrm{~s}^{2}$} \\
\hline 76 & Osmio & Os & {$[\mathrm{Xe}] 4 \mathrm{f}^{14} 5 \mathrm{~d}^{6} 6 \mathrm{~s}^{2}$} \\
\hline 77 & Iridio & Ir & {$[\mathrm{Xe}] 4 \mathrm{f}^{14} 5 \mathrm{~d}^{7} 6 \mathrm{~s}^{2}$} \\
\hline 78 & Platino & $\mathrm{Pt}$ & {$[\mathrm{Xe}] 4 \mathrm{f} 145 \mathrm{~d} 96 \mathrm{~s} 1$} \\
\hline 79 & Oro & $\mathrm{Au}$ & {$[\mathrm{Xe}] 4 \mathrm{f}^{14} 5 \mathrm{~d}^{10} 6 \mathrm{~s}^{1}$} \\
\hline 80 & Mercurio & $\mathrm{Hg}$ & {$[\mathrm{Xe}] 4 \mathrm{f}^{14} 5 \mathrm{~d}^{10} 6 \mathrm{~s}^{2}$} \\
\hline 81 & Talio & $\mathrm{Tl}$ & {$[\mathrm{Xe}] 4 \mathrm{f}^{14} 5 \mathrm{~d}^{10} 6 \mathrm{~s}^{2} 6 \mathrm{p}^{1}$} \\
\hline 82 & Plomo & $\mathrm{Pb}$ & {$[\mathrm{Xe}] 4 \mathrm{f}^{14} 5 \mathrm{~d}^{10} 6 \mathrm{~s}^{2} 6 \mathrm{p}^{2}$} \\
\hline 83 & Bismuto & $\mathrm{Bi}$ & {$[\mathrm{Xe}] 4 \mathrm{f}^{14} 5 \mathrm{~d}^{10} 6 \mathrm{~s}^{2} 6 \mathrm{p}^{3}$} \\
\hline 84 & Polonio & Po & {$[\mathrm{Xe}] 4 \mathrm{f}^{14} 5 \mathrm{~d}^{10} 6 \mathrm{~s}^{2} 6 \mathrm{p}^{4}$} \\
\hline 85 & Astato & At & {$[\mathrm{Xe}] 4 \mathrm{f}^{14} 5 \mathrm{~d}^{10} 6 \mathrm{~s}^{2} 6 \mathrm{p}^{5}$} \\
\hline 86 & Radón & $\mathrm{Rn}$ & {$[\mathrm{Xe}] 4 \mathrm{f}^{14} 5 \mathrm{~d}^{10} 6 \mathrm{~s}^{2} 6 \mathrm{p}^{6}$} \\
\hline 87 & Francio & $\mathrm{Fr}$ & {$[\mathrm{Xe}] 4 \mathrm{f}^{14} 5 \mathrm{~d}^{10} 6 \mathrm{~s}^{2} 6 \mathrm{p}^{6} 7 \mathrm{~s}^{1}$} \\
\hline 88 & Radio & $\mathrm{Ra}$ & {$[\mathrm{Xe}] 4 \mathrm{f}^{14} 5 \mathrm{~d}^{10} 6 \mathrm{~s}^{2} 6 \mathrm{p}^{6} 7 \mathrm{~s}^{2}$} \\
\hline 89 & Actinio & Ac & {$[\mathrm{Xe}] 4 \mathrm{f}^{14} 5 \mathrm{~d}^{10} 6 \mathrm{~s}^{2} 6 \mathrm{p}^{6} 6 \mathrm{~d}^{1} 7 \mathrm{~s}^{2}$} \\
\hline
\end{tabular}

Tabla 2. Configuración electrónica de los principales elementos químicos

Existen dos tipos de muones, el muón $\left(\mu^{-}\right)$y el antimuón $\left(\mu^{+}\right)$, compartiendo similitudes con el electrón y el positrón, pero siendo más pesados. Las principales características son (Aloy, 2017):

\begin{tabular}{|c|c|c|c|}
\hline Símbolo & Carga & $\begin{array}{c}\text { Masa (con respeto } \\
\text { al protón) }\end{array}$ & $\begin{array}{c}\text { Tiempo de vida en } \\
\text { segundo }\end{array}$ \\
\hline$\mu^{+}$ & +1 & $1 / 9$ & $10^{-6}$ \\
\hline$\mu^{-}$ & -1 & $1 / 9$ & $10^{-6}$ \\
\hline
\end{tabular}

Tabla 3. Características del muón 
Al ser una partícula de mayor masa que el electrón, al desintegrarse produce (frecuentemente) un electrón libre, un antineutrino electrónico y un neutrino muónico, cuya carga eléctrica total es nula. El muón es un "electrón gigante" pero altamente inestable, por ello, "busca" convertirse en una partícula más ligera y por lo tanto estable, así (Díaz, 2015):

$$
\mu \longrightarrow e+\bar{V} e+V \mu
$$

El leptón tau (a veces llamado tauónico), fue observado por primera vez en 1975 por Martin Lewis Pearl, y es el más pesado de las partículas leptónicas, la cual posee una vida media de $3,3 \times 10^{-3}$ segundos, su masa es de aproximadamente $1777 \mathrm{MeV} / \mathrm{c}^{2}$, es decir, dos veces más grande que el protón y unas 3498 más que el electrón (Perl, 2004).

La partícula tau se origina por la colisión de un electrón y un positrón a la velocidad de la luz. Inicialmente se la consideraba como un mesón, ya que se requería los mismos niveles de energía para crearse, posteriormente se determinó que se trataba de una partícula diferente. El proceso colisionador de electrón-positrón, para crear la partícula de tau es el siguiente (Perl, 2004):

$$
e^{+}+e^{-} \longrightarrow \tau^{+}+\tau^{-} \longrightarrow e^{ \pm}+\mu^{ \pm}+\operatorname{Ve}\left(\text { si } e^{+}\right)
$$

Al igual que los otros leptones, el tau cumple la simetría de conjugación de carga, inversión de paridad e inversión temporal (CPT). Esta partícula al tener una masa muy alta, es capaz de desintegrase en hadrones, según las siguientes proporciones:

- $18 \%$ de las veces se forma un electrón, dos neutrinos y un neutrino tauónico

- $18 \%$ de las veces se forma un muón, dos neutrinos y un neutrino tauónico

- $64 \%$ de las veces se forma hadrones, un neutrino y un neutrino tauónico

Los primeros pasos para el descubrimiento de los neutrinos, fueron derivados de los estudios de la radioactividad. A comienzos del siglo XX, se habían definido tres tipos de radiación, la alfa $(\alpha)$, la beta $(\beta)$ y la gamma $(\gamma)$, cuyas propiedades podían ser analizadas, pero no tenían un fundamento teórico que las respaldase. Donde la desintegración $\beta$ era definida como el proceso natural en el cual ciertos elementos químicos (con radiactividad), se transforman en otros, cuyo producto adicional es el tipo de radiactividad $\beta$ (radiación en forma de flujos de electrones). En 1914 se determinó que el espectro de la radiación $\beta$ es continuo (siendo esto un problema que amenazaba la ley de la conservación de la energía), lo que difería con los espectros de radiación $\alpha$ y $\gamma$, los cuales eran discretos; ya que Bohr en 1913, determinó que los átomos tenían energía cuantificada en valores discretos y determinados, por ello, la radiación emitida de estos, también debía ser 
similar, sin embargo, los experimentos mostraban que poseía un espectro continuo(Cassini, 2012).

Este dilema, hizo que varios científicos propusieran repostular el principio de la conservación de la energía. La respuesta a esta interrogante vino de la mano de Wolfgang Ernst Pauli en 1930, quien propuso que conjuntamente a la partícula $\beta$, se debería estar emitiendo (de manera no detectable) otra partícula adicional, la misma que no posee carga eléctrica, llevándose parte de la energía y que por su neutralidad no podía ser revelada. Los neutrinos siguen la siguiente ecuación (Bonzi, 2015):

$$
n \longrightarrow p^{+}+e^{-}+V e
$$

En 1932, el inglés James Chadwick descubre el neutrón, los cuales eran masivamente superiores a las partículas hipotéticas de Pauli, por ello este último científico decidió nombrarlas como neutrinos, para diferenciarlos de los neutrones pesados. Entre las propiedades que se le dio a los neutrinos estaban: su masa debía ser mucho más pequeña a la de los electrones (Perrin, 1933), la interacción con la materia es casi nula (Ernst, 1934).

En 1956, los científicos Frederick Reine y Clyde Lorrain Cowan Jr., logran detectar a los neutrinos (electrónicos) a través de la relación señalruido; por su parte Reines y Cowan en 1953 realizan el siguiente experimento para encontrar a estas partículas:

Usando un detector que constaba de un blanco de 400 litros de agua y cloruro de cadmio. El antineutrino producido en el reactor nuclear es emitido e interactúa con un protón del detector, produciendo un positrón y un neutrón. El positrón se aniquila con un electrón del material emitiendo simultáneamente dos fotones y el neutrón con baja energía cinética (neutrón térmico) es capturado eventualmente por un núcleo de cadmio, produciendo una emisión de fotones aproximadamente 15 microsegundos después de la aniquilación del positrón. El proceso observado es el inverso del decaimiento beta conocido (Bonzi, 2015).

Los neutrinos muónicos $(V \mu)$ y tauónicos $(V \tau)$, son esquivos en su detección, por lo tanto permanecen siendo netamente teóricos, donde se ha podido establecer que su masa es 200.000 veces más pequeñas que las del electrón. La mayoría de estas partículas (neutrinos) derivan del Big Bang, los cuales forma la radiación de fondo cósmica (Campo, Ardid, Tortosa, Felis, \& Martínez, 2017).

Para la detección de estos neutrinos se han diseñado telescopios submarinos, que miden la interacción lumínica de los neutrinos en un medio denso (agua o hielo), la cual es potenciada en los fotomultiplicadores repartidos en grandes estructuras que forman arreglos lineales. Al interactuar los neutrinos con el medio produce una cascada de hadrones, del cual, el $25 \%$ de la energía es depositada en un pequeño volumen, provocando que aumente 
la temperatura, y con ello se genera un pulso acústico de forma bipolar de varios $\mathrm{kHz}$ (Felis, 2017).

Los neutrinos pueden pasar de una familia a otra, a este proceso se lo llama oscilación de neutrinos, quizás por ello siguen siendo esquivos en su detección. Se creen que además de los neutrinos electrónicos, muónicos y tauónicos, existen dos tipos de neutrinos adicionales, llamados neutrinos estériles, los cuales no interactúan con la materia de ninguna forma, sino únicamente con la gravedad (Kopp, Maltoni, \& Schwetz, 2011).

La importancia de estudiar las propiedades de los neutrinos (principalmente la masa), radica en que estos son las partículas más abundantes del universo, además tienen una interacción débil con la demás materia, por ello, estas partículas pueden mostrar una radiografía completa del universo desde el primer momento en que se formó (el Big Bang), ya que estos "neutrinos remantes", aún se encuentran en las regiones más lejanas del horizonte del espacio (Zeleny, 2015).

\section{Los bosones}

Los bosones son las partículas que trasmiten la fuerza resultante entre la interacción de las partículas (también se los denomina como bosones de gauge). Las 4 fuerzas que se transmiten son el electromagnetismo, la fuerza nuclear fuerte y débil, y la gravedad, donde cada una de estas fuerzas tiene su independiente partícula de transmisión. El fotón, es el encargado de transmitir la fuerza electromagnética entre dos partículas cargadas eléctricamente, en otras palabras, esta partícula constituye la mínima expresión de la luz. La fuerza nuclear fuerte tiene como partícula transmisora al denominado gluón (mantiene unidos a los quarks dentro del núcleo atómico), este nombre deriva de la palabra inglesa "glue", que significa pegamento. La fuerza nuclear débil, tiene dos partículas mediadoras llamadas bosón W y bosón Z. Hipotéticamente el gravitón es el bosón que transmite la gravedad, sin embargo esta conjetura no ha sido demostrada, por lo cual, en algunos modelos está excluida. Finalmente el bosón de Higgs es la partícula que otorga de masa a todas las partículas detallas por el modelo estándar (Reina, 2005). 


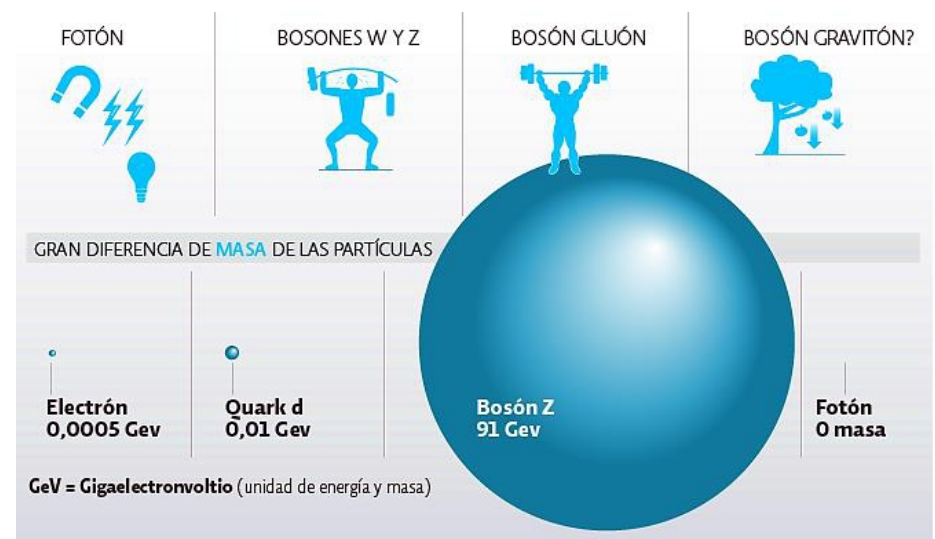

\section{Ilustración 3. Diagrama de los efectos de las partículas bosónicas. Fuente: Agence France-Presse (AFP), El Comercio, 2012}

Los bosones poseen un espín entero equivalente a 0,1 y 2 , además no cumplen con el principio de exclusión de Paulli, ni siguen las estadísticas de Fermi y Dirac, ya que se basan en las estadísticas de Bose-Einstein (estadísticas aplicadas a este grupos grandes de partículas elementales, las cuales son capaces de coexistir en el mismo estado cuántico y con el mismo equilibrio térmico). Los bosones pueden ser ser creados sin limitaciones fabricados en grandes cantidades, donde el número de fermiones resultantes es constante, es decir su cantidad se conserva en las interacciones (Cadenas, 2004).

Los fotones y los gluones no tienen masa ni carga eléctrica, sin embargo los gluones poseen una carga de color dependiente al color de los quarks. Los bosones $\mathrm{W}$ pueden tener carga eléctrica positiva o negativa (siendo una la antipartícula de la otra), con una masa de 80,4 GeV $(1 \mathrm{GeV}=$ $1000 \mathrm{MeV}$ ); el bosón $\mathrm{Z}$ no posee carga eléctrica, pero es altamente masivo con unos 91,19 GeV de masa. El bosón de Higgs tampoco dispone de una carga eléctrica o de sabor, pero tiene la mayor masa de todos los bosones de aproximadamente $125 \mathrm{GeV}$ (Seltzer, 1993; Tao, 2006; Abachi, et al., 1996; Khachatryan, et al., 2015).

El resumen de las características de los leptones es la siguiente:

\begin{tabular}{|c|c|c|c|c|}
\hline Nombre & Símbolo & Masa $\left(\mathbf{G e V} / \mathbf{c}^{2}\right)$ & Carga eléctrica (e) & Espín \\
\hline Fotón & $\gamma$ & 0 & 0 & 1 \\
\hline Gluón & $g$ & 0 & $\begin{array}{c}0 \text { (posee carga de } \\
\text { color octeta) }\end{array}$ & 1 \\
\hline Bosón $\mathrm{W}$ & $W$ & 80,401 & \pm 1 & 1 \\
\hline Bosón Z & $Z$ & 91,1876 & 0 & 1 \\
\hline Gravitón & $G$ & $2,9 \times 10^{-21} \mathrm{eV}(*)$ & No determinada & 2 \\
\hline Higgs & $H$ & $125,3 \pm 0.4$ & 0 & 0 \\
\hline \multicolumn{5}{|c}{ (*Zakharov, Jovanovic, Borka, \& Jovanovic, 2017) } \\
\hline
\end{tabular}

Tabla 4. Detalle de las diferentes características de los bosones 
A finales del siglo XIX, existían varias teorías con vacíos en el marco fundamentado por la mecánica y la electrodinámica, entre ellas se encontraba la radiación que emiten y absorben ciertos materiales, donde científicos como Maxwell lo interpretaban como el movimiento oscilatorio de las cargas eléctricas que emiten energía de forma constante cuyo resultado son las ondas electromagnéticas, siendo estas, afirmaciones incorrectas (Arons, 1997).

En 1960 Kirchhof estudió la uniformidad resultante de la radiación emitida por un objeto (independiente de su composición), la cual es exclusivamente por la temperatura, existiendo un espectro característico y una intensidad variable con la frecuencia, pero fue Planck en 1900 quien propuso que la energía de un cuerpo era proporcional a su frecuencia $(\varepsilon=h . v)$, donde un cuerpo estaría formado por pequeños elementos a los cuales llamó resonadores que vibraban a determinadas frecuencias, además podría existir intercambio de energía en cantidades finitas llamadas "cuantos". El intercambio discontinuo de la energía, parecía no calar en la mente de algunos científicos (dada la falta de postulados que llegarían posteriormente), ya que se consideraba que los procesos que realizaba la naturaleza siempre debían ser continuos, y de esta forma mantener la relación causa-efecto, para utilizar métodos matemáticos como el cálculo integral y el diferencial, los cuales daban resultados alineados a la conservación de la energía. Sin embargo, los nuevos experimentos confirmaban la validez de la ley de Planck, así que los físicos tuvieron que considerar el diseño de una física discontinua que explicara componentes aleatorios, donde el mismo Einstein en 1905, propuso la cuantización de la energía de un oscilador, posteriormente se sumaron Eherenfest, Laue, Jeans, Lorentz, entre otros (Kuhn, 1987).

Einstein se sentía frustrado por no ser capaz de entender que eran los cuantos de luz (tal como lo indica su autobiografía), ya que inicialmente no le atribuía un carácter corpuscular a los mismos, no obstante, años después empezaba a considerar dicha posibilidad, dándola atributos semicorpusculares a su teoría de 1905 (de la relatividad especial). La propuesta de Einstein modificaba de manera profunda la concepción de la radiación, ya que en esta época, la misma tenía propiedades ondulatorias y poseían valores continuos para la energía, en la teoría del efecto fotoeléctrico, en la cual la conservación de cantidad de momento y energía se computa como si rayos $\mathrm{X}$ fuesen entes corpusculares. En 1915 con los experimentos de Millikan y de forma definitiva en 1923 con los resultados de Compton y su equipo, los cuantos son considerados de carácter corpuscular (Savall, Doménech, \& Martínez, 2013).

$\mathrm{El}$ experimento de Compton, pretendía demostrar que los rayos $\mathrm{X}$ eran otro tipo de radiación electromagnética, cuyo comportamiento sería similar al de la luz visible (refracción, reflexión especular, difracción, etc.), donde analizaron la dispersión de los rayos $\mathrm{X}$ (por efectos de los electrones) en una superficie de carbono, y se observó que la longitud de onda de estos rayos 
(resultantes) eran superiores a la de los rayos incidentes (iniciales). Compton sugirió que este efecto puede ser explicado si se considera a la luz como un haz de corpúsculos (cuantos), los cuales no tienen masa pero si un momento lineal de $h v / c$; donde uno de los cuantos incidentes $\left(h v_{0}\right)$ es absorbido por uno de los electrones centrales, los cuales emiten un segundo cuanto de menor energía $(h v)$, pero con menor frecuencia y mayor longitud de onda de acuerdo a la siguiente fórmula:

$$
\lambda_{f}-\lambda_{i}=\Delta \lambda=\frac{h}{m_{e} c}(1-\cos \theta)
$$

Dónde: $\lambda_{f}$ son los rayos $\mathrm{X}$ resultantes, $\lambda_{i}$ son los rayos $\mathrm{X}$ iniciales, $h$ es la constante de Planck, $m_{e}$ es la masa de la partícula (electrones), y $c$ es la velocidad de la luz.

Teniendo como consecuencia que el electrón sea dispersado con una energía de $h\left(v_{0}-v\right)$, donde la energía y el momento se conservan en el evento de colisión entre el fotón y el electrón. En 1926 en científico Lewis introdujo al vocabulario de los físicos la palabra fotón (de la palabra griega phos que significa luz) en su artículo titulado "The nature of light", en el que mencionaba la hipotética simetría de los fenómenos de emisión y absorción de luz por los átomos. En la década de los 30, existían al menos dos teorías de la luz, una sostenía que tenía propiedades ondulatorias y la otra que la luz tenía características corpusculares; y para Einstein, estos dos modelos eran imprescindibles, pero sin conexión lógica (Savall, Doménech, \& Martínnez, 2013).

El francés Louis De Broglie en 1924, en su tesis doctoral propuso la dualidad de la luz (con su partícula el fotón), la cual presenta propiedades ondulatorias como corpusculares (en la actualidad se la denomina dualidad onda-corpúsculo), comportándose como onda en la refracción, difracción, polarización o en las interferencias luminosas, y se comporta como una partícula cuando interactúa con la materia, por ejemplo en la colisión entre fotones, electrones, protones, etc. (transferencia fija de energía). Estas aportaciones sirvieron para unificar dos fenómenos para el mismo objeto de estudio (la luz), y es el fundamento de la mecánica cuántica en la descripción de los fenómenos atómicos (Segura, Nieto, \& Segura, 2012).

Los fotones son emitidos en los procesos naturales, no pueden desintegrarse en el vacío, además poseen dos estados de polarización, los cuales pueden ser descritos a través de tres parámetros sucesivos o continuos (vector y longitud de onda y dirección de propagación), su velocidad en el vacío equivale a la velocidad de la luz $\left(\approx 3.10^{6} \mathrm{~m} / \mathrm{seg}\right)$, que siguen un momento lineal (Spavieri, Erazo, \& Sánchez, 2009).

La fuerza mediadora de los quarks, son los ocho campos vectoriales llamados gluones, quienes no poseen una masa definida. Los quarks cambian de color cuando se intercambian gluones, por ello, la carga final del sistema 
es siempre la misma (antes y después de la emisión o adsorción). Los quarks y los gluones conforman a los hadrones, quienes no tienen carga de color neta, por la propiedad del "confinamiento", la cual no ha sido explicada en su totalidad por la teoría cuántica. Por otro lado, existe la propiedad de acoplamiento, definida como la constante entre la interacción de quarks y gluones, la misma que disminuye a medida que la energía del sistema aumenta (también denominada libertad asintótica), donde eventualmente estos dos partículas conforman un plasma (Gorrin, 2018).

Los bosones $\mathrm{W}$ y $\mathrm{Z}$ entran en interacción cuando un leptón o un quark cambian de sabor, para convertirse en partículas más ligeras, de tal forma que intervienen otros tres tipos de bosones intermedios. Estos bosones estan presentes en la desintegración beta, cuando un neutrón se transforma en un protón, produciéndose adicionalmente un electrón y un electrón antineutrino, esta transformación solo es posible ya que un quark down se convierte en un quark up, y con el decaimiento del bosón W (negativo para mantener la carga del sistema) en dos leptones que permiten la emisión del electrón y del neutrino. Por su parte el bosón Z, actúa como partícula portadora del momento lineal, el cual se lo considera como intermediario de un nuevo tipo de reacción entre los neutrinos. Esta llamada "interacción de corriente neutral" fue la pieza faltante de un rompecabezas en el que las fuerzas creadas por los bosones W se unen perfectamente con la fuerza del electromagnetismo, debido al fotón (Miransky, Tanabashi, \& Yamawaki, 1989).

La gravedad ha generado debates vigorosos sobre el concepto de masa en reposo de gravitones. Según la Relatividad General, la gravedad es un fenómeno de la curvatura (de Lorentz) del espacio-tiempo de una determinada región en el universo (variando según las condiciones), donde el espaciotiempo es un lugar geométrico de cuatro dimensiones $\left(\mathrm{x}_{0}, \mathrm{x}_{1}, \mathrm{x}_{2}, \mathrm{x}_{3}\right)$, donde es tangencial al plano Minkowski (Guillén, 2006). De acuerdo con las propiedades del campo gravitatorio, el gravitón debe ser un bosón de espín par (2); mientras que la masa, según mediciones experimentales, dan una medida aproximada de $1,6 \times 10^{-69} \mathrm{~kg}$, aunque es posible que sea igual a cero (Gershtein, et al., 2003). Existe un formalismo altamente desarrollado para tratar de medir las desviaciones de la gravedad, ya que en este marco, se han realizado muchas mediciones, principalmente en condiciones de campo débil, tanto para fenómenos de baja velocidad como de alta velocidad (para detectar desviaciones), donde ninguna de estas mediciones hasta la fecha, ha producido resultados "inesperados", solo límites cada vez más estrictos para las desviaciones de la gravedad de Einstein (Goldhaber \& Nieto, 2010).

En el año 2012 se anunció el descubrimiento del bosón de Higgs de parte de los científicos del CERN (Organización Europea para la Investigación Nuclear, en español) en colaboración de científicos de todo el mundo, marcando así un hito histórico para el mundo académico, ya que finalmente se 
encontraba al responsable de la masa de toda la materia que conforma el universo. Este bosón rompe la simetría espontánea a través del mecanismo del campo de Higgs, de la siguiente manera:

El campo de Higgs en el modelo estándar actúa en forma similar a la fuerza que elonga al resorte hasta exceder su límite de elasticidad rompiendo su simetría inicial. El campo de Higgs actúa sobre el campo electrodébil llevándolo de un estado simétrico de una determinada energía a un estado antisimétrico de una energía menor (energía mínima). El bosón de Higgs tal y como es visto en el modelo estándar es una vibración del campo de Higgs (Valencia \& Bernal, 2016).

\section{Conclusiones}

El actual modelo estándar agrupa a 18 partículas, agrupadas en los denominados: fermiones (quarks y leptones) y bosones (de gauge).

Los quarks y los leptones son fundamentalmente las partículas elementales, ya que no poseen otras partículas en su estructura interna (como el caso de los quarks).

Existen 6 sabores o tipos de quarks: up, down, strange, charm, bottom y top. Los quarks son los constituyentes que conforman el núcleo atómico (neutrones y protones).

Existen 6 leptones: electrón, muón, tauón, el neutrino del electrón (positrón), el neutrino del muón y el neutrino del tauón. El neutrino es una de las partículas con mayor relevancia, tanto para físico como astrónomos, los cuales consideran que esta partícula puede generar una "fotografía" del universo en los primeros instantes del Big Bang.

Existen 6 bosones: fotón, gluón, bosón W, bosón Z, gravitón y bosón de Higgs. Los bosones son las partículas mediadoras de las 4 fuerzas dan orden al universo (electromagnetismo, fuerza nuclear fuerte, fuerza nuclear débil y la gravedad, esta última aún se encuentra en análisis del modelo estándar).

\section{References:}

1. Abachi, S., Abbott, B., Abolins, M., Acharya, B., Adam, I., Adams, D., \& Alvarez, G. (1996). Measurement of the W boson mass. Physical ReviewLetters, 77(16), 3309.doi:https://doi.org/10.1103/PhysRevLett.77.3309

2. Aloy, M. (2017). Incorporación de la física contemporánea en el nivel medio de la educación formal. La Plata, Argentina: Universidad Nacional de La Plata. Obtenido de http://sedici.unlp.edu.ar/bitstream/handle/10915/61037/Documento_c ompleto__.pdf-PDFA1b.pdf? sequence $=1$ \&isAllowed $=y$ 
3. Amsler, C., DeGrand, T., \& Krusche, B. (2018). Quark Model. Obtenido de http://pdg.lbl.gov/2019/reviews/rpp2018-rev-quarkmodel.pdf

4. Arons, A. (1997). Teaching introductory physics. New York: John Wiley \& Sons.

5. Aviléz, M. (2013). El modelo estándar de partículas. Obtenido de https://fisquiweb.es/Apuntes/Apuntes2Fis/ModeloEstandar.pdf

6. Bonzi, E. (2015). Neutrinos. Revista de Enseñanza de la Física, 27(2), 41-50.

7. Botella, F., Branco, G., Rebelo, M., \& Silva-Marcos, J. (2016). What if the masses of the first two quark families are not generated by the standard model Higgs boson? Physical Review, 94(11), 1-6. doi:10.1103/PhysRevD.94.115031

8. Cabarcas, J. (2009). Física más allá del Modelo Estándar y su posible observación experimental (tesis de doctorado). La Plata, Argentina: Universidad Nacional de la Plata. doi: https://doi.org/10.35537/10915/2612

9. Cadenas, Y. (2004). Espistemología, ontología y complementariedad en Niels Bohr. Madrid, España: Universidad Complutense de Madrid. Obtenido de https://eprints.ucm.es/5274/1/T27024.pdf

10. Callister, W. (2007). Introducción a la ciencia e ingeniería de los materiales. Barcelona, España: Reverté S.A.

11. Campo, M., Ardid, R., Tortosa, D., Felis, E., \& Martínez, J. (2017). Estudio del efecto paramétrico en agua con aplicación a un calibrador acústico para detección de neutrinos. Obtenido de Sea-acustica: http://www.sea-acustica.es/fileadmin/Coruna17/ULT-0\%20021.pdf

12. Cassini, A. (2012). La invención del neutrino: un análisis epistemológico. Scientiae Studia, 10(1), 11-39. doi:http://dx.doi.org/10.1590/S1678-31662012000100002

13. Cobián, J. (2018). El modelo estandar de la física de partículas. Sociedad Nuclear Española, 1-13.

14. Díaz, G. (2015). Determinación del error sistemático del momentum de muones producidos por interacciones neutrino-nucleón en el detector MINERVA. Perú: Pontificia Universidad Católica del Perú. Obtenido de http://lss.fnal.gov/archive/masters/fermilab-masters2015-09.pdf

15. El Comercio (2012). Descubren una partícula coherente con el bosón de Higgs (infografía). Obtenido de: https://especiales.elcomercio.com/infografias/2012/07/particuladedio s/Index.html 
16. Felis, E. (2017). Tecnologías acústicas para la detección de Materia Oscura. Diseño y desarrollo de un detector Geyser. Valencia, España: Universitat Politècnica de València. doi:10.4995/Thesis/10251/86282.

17. Galicia, H. (2014). Contribución de partículas exóticas al decaimiento del bosón de Higgs a dos fotones. Puebla, México. Obtenido de Benemétrica Universidad Autónoma de Puebla: https://www.fcfm.buap.mx/assets/docs/docencia/tesis/fisica/2014/Gal iciaSantosHelenaGabriela.pdf

18. Gelmeni, G. (2014). El boson de Higgs. Ciencia e Investigación, 64(3), 1-19.

Obtenido

de https://arxiv.org/ftp/arxiv/papers/1407/1407.1741.pdf

19. Gershtein, S., Logunov, A., Mestvirishvili, M., \& Tkachenko, N. (2003). Graviton Mass, Quintessence, and Oscillatory Character of Universe Evolution. Elementary Particles and Fields Theory, 67(8), 1596-1604.

20. Goldhaber, A., \& Nieto, M. (2010). Photon and Graviton Mass Limits.
Reviews
of
Modern
Physics,
82 ,
939-979.

doi:10.1103/RevModPhys.82.939

21. Gorrin, D. (2018). Física de hadrones y de plamas de quarks y gluones desde la dualidad AdS/CFT. La Plata, Argentina: Universidad Nacional de La Plata. Obtenido de http://sedici.unlp.edu.ar/bitstream/handle/10915/78526/Documento_c ompleto.pdf-PDFA.pdf?sequence $=1$ \&isAllowed $=\mathrm{y}$

22. Guillén, A. (2006). La velocidad de la gravedad. Ciencia Abierta, 7(79).

Obtenido

de

https://www.researchgate.net/profile/Alfonso_Leon_Guillen_Gomez/ publication/200123152_La_velocidad_de_la_gravedad/links/06deb88 dcbecefa3aea88a0b/La-velocidad-de-la-gravedad.pdf

23. Hendry, A., \& Lichtenberg, D. (1978). The quark model. Reports on Progress in Physics, 41, 1707-1780. doi:10.1088/00344885/41/11/001

24. Ji, X. (1995). A QCD analysis of the mass structure of the nucleon. Physical Review Letters, 74(7), 1-9. doi:https://doi.org/10.1103/PhysRevLett.74.1071

25. Khachatryan, V., Sirunyan, A., Tumasyan, A., Adam, W., Bergauer, T., Dragicevic, M., . . . Jeitler, M. (2015). Precise determination of the mass of the Higgs boson and tests of compatibility of its couplings with the standard model predictions using proton collisions at 7 and $8 \mathrm{TeV}$. The European Physical Journal C, 75(5), 212.

26. Kopp, J., Maltoni, M., \& Schwetz, T. (2011). Are There Sterile Neutrinos at the eV Scale? Physical Review Letters, 107(9), 1-5. doi:https://doi.org/10.1103/PhysRevLett.107.091801 
27. Kuhn, T. (1987). La Teoría del Cuerpo Negro y la Discontinuidad Cu'antica, 1894-1912. Madrid: Alianza Editorial.

28. Meyer, H. (2011). Hadron structure on the Lattice. The European Physical Journal Special Topics, 49(198), 1-17. doi:10.1140/epjst/e2011-01482-3

29. Mileo, N. (2017). Estudio fenomenológico de extensiones del sector escalar del modelo estándar. Obtenido de Repositorio institucional de la UNLP: http://inspirehep.net/record/1605066/files/fulltext.pdf

30. Miransky, V., Tanabashi, M., \& Yamawaki, K. (1989). Is the t quark responsible por the mass of $\mathrm{W}$ and $\mathrm{Z}$ bosons? Modern Physics Letters A, 4(11), 1043-1053. doi:10.1142/S0217732389001210

31. Moreira, M. (2007). La física de los quarks y la epistemología. Revista Brasileira de Ensino de Física, 29(2), 1-13.

32. Moreira, M. (2009). El modelo estándar de la física de partículas. Revista Brasileña de Enseñanza de Física, 31(1), 1-16.

33. Ordoñéz, M. (1992). Partículas elementales. Física Nuclear \& Partículas, 4, 71-75.

34. Perl, M. (2004). The discovery of the tau lepton and the changes in elementary-particle physics in forty years. Physics in Perspective, 6(4), 401-427. doi:https://doi.org/10.1007/s00016-003-0218-3

35. Physicsmore. (2019). Teoría de cuerdas (la ciencia de las supercuerdas). Obtenido de https://physicsmore.blogspot.com/search/label/modelo $\% 20$ est $\%$ C $3 \%$ A1ndar

36. Reina, D. (2005). ¿Partícula inmortal? ¿Cómo ves? (84), 16-19. Obtenido de http://www.comoves.unam.mx/assets/revista/84/guiadelmaestro_84.p df

37. Rivas, M. (2016). Teoría Cinemática de partículas elementales con espín. Bilbao: Universidad del País Vasco.

38. Sánchez, R. (2014). Propiedades electromagnéticas de un neutrino en el modelo más simple con un bosóon de Higgs ligero. Puebla: Benemérita Universidad Autónoma de Puebla. Obtenido de https://www.fcfm.buap.mx/assets/docs/docencia/tesis/fisica/2014/San chezVelezRicardo.pdf

39. Savall, F., Doménech, J., \& Martínez, J. (2013). La introducción del concepto de fotón en bachillerato. Revista Brasileira de Ensino de Fíisica, 35(2), 1-14.

40. Segura, A., Nieto, V., \& Segura, E. (2012). Un análisis profundo del fenómeno dualidad onda-partícula para la comprensión del mundo cuántico. Latin-American Journal of Physics Education, 6(1), 137-142. 
41. Seltzer, S. (1993). Calculation of Photon Mass Energy-Transfer and Mass Energy-Absorption Coefficients. Radiation Research, 136(2), 147-170. doi: $10.2307 / 3578607$

42. Silva, P. (2011). Analysis of the mass structure of the hadrons. Physics, 1-15. Obtenido de https://arxiv.org/abs/1108.2073

43. Spavieri, G., Erazo, J., \& Sánchez, A. (2009). Momento electromagnético, efectos cuánticos y la masa del fotón. Revista Mexicana de Física, 55(4), 236-248.

44. Tao, W. (2006). Particle Data Group. Journal of Physics G: Nuclear and Particle Physics, 33(1), 1232. doi:10.1088/0954-3899/33/1/001

45. Universidad de Navarra. (2012). Grupo Ciencia, Razón y Fe. Obtenido de https://www.unav.edu/web/ciencia-razon-y-fe/el-modelo-standardde-particulas-elementales

46. Valencia, J., \& Bernal, J. (2016). Un modelo didáctico para comprender el bosón de Higgs como parte del modelo estándar de partículas elementales. Ciencia Ergo Sum, 23(2), 163-170.

47. Villatoro, F. (2012). La masa de un protón, la masa de sus quarks y la energía cinética de sus gluones. Obtenido de https://francis.naukas.com/2012/04/30/la-masa-de-un-proton-lamasa-de-sus-quarks-y-la-energia-cinetica-de-sus-gluones/

48. Zakharov, A., Jovanovic, P., Borka, D., \& Jovanovic, V. (2017). Different ways to estimate graviton mass. International Journal of Modern Physics: Conference Series, 47, 1-6. doi:10.1142/S2010194518600960

49. Zeleny, M. (2015). Cálculo y análisis estadístico de los ángulos de mezcla de neutrinos en el THDM-III. Puebla, México: Benemérita Universidad Autónoma de Puebla. Obtenido de https://www.fcfm.buap.mx/assets/docs/docencia/tesis/fisica/2015/Zel enyMoraMoises.pdf 